D'après ce que nous avons démontré la fonction $\mathrm{F}$ a les valeurs suivantes :

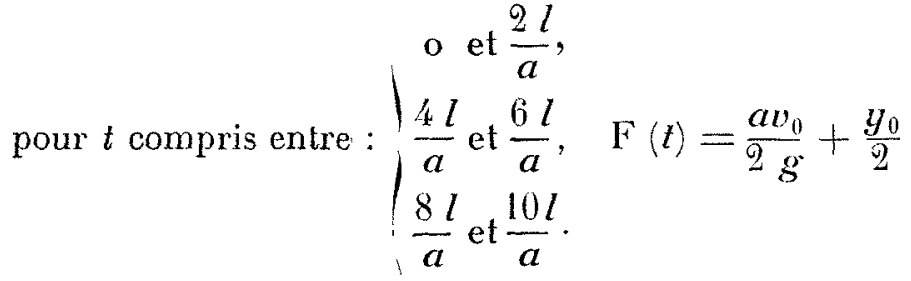

$$
\begin{aligned}
& \text { pour } t \text { compris entre }:\left\{\begin{array}{l}
\frac{2 l}{a} \text { et } \frac{4 l}{a}, \\
\frac{6 l}{a} \text { et } \frac{8 l}{a}
\end{array} \quad \mathrm{~F}(t)=\frac{a v_{0}}{2 g}-\frac{y_{0}}{2}\right.
\end{aligned}
$$

D’ailleurs, en un point quelconque $\mathrm{M}$ de la conduite sitùé à une distance $x$ de l'extrémité avall, on a $\mathrm{AM}=x$.

$$
y=y_{0}+\mathrm{F}\left(t-\frac{x}{a}\right)-\mathrm{E}\left(t-\frac{2 l}{a}+\frac{x^{\prime}}{a}\right) \text {. }
$$

Etudions par exemple le graphique des pressions en $\mathrm{C}$, premier tiers amont. Le manomètre est placé en $\mathrm{C}$ :

$$
\mathrm{AC}=\frac{2 l}{3}
$$

on a :

époque $\frac{2 l}{a}, y=y_{0}+\mathrm{F}\left(\frac{2 l}{a}-\frac{2 l}{3 a}\right)-\mathrm{F}\left(\frac{2 l}{a}-\frac{2 l}{a}+\frac{2 l}{3 a}\right)=y_{0}$ époque $\frac{3 l}{a}, y=y_{0}+\mathrm{F}\left(\frac{3 l}{a}-\frac{2 l}{3 a}\right)-\mathrm{F}\left(\frac{3 l}{a}-\frac{2 l}{a}+\frac{2 l}{3 a}\right)=0$ époque $\frac{4 l}{a}, y=y_{0}+\mathrm{F}\left(\frac{4 l}{a}-\frac{2 l}{3 a}\right)-\mathrm{F}\left(\frac{4 l}{a}-\frac{2 l}{a}+\frac{2 l}{3 a}\right)=y_{0}$ époque $\frac{5 l}{a}, y=y_{0}+\mathrm{F}\left(\frac{5 l}{a}-\frac{2 l}{3 a}\right)-\mathrm{F}\left(\frac{5 l}{a}-\frac{2 l}{a}+\frac{2 l}{5 a}\right)=2 y_{0}$ Le cliché $\mathbf{5} 5$ donne l'inscription de la pression, en $c$, au moyen du graphique i 5 et du graphique correspondant pris à l'extrémité aval, on peut, en distinguant les secondes par un signal, vérier la concordance des' phases.

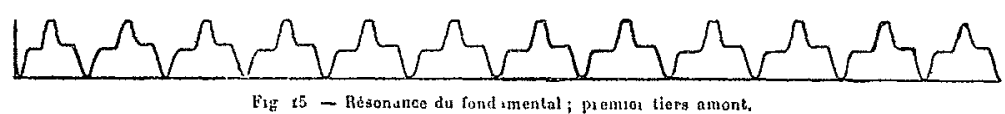

Fig. I6 - Resonance de l'narmonque 3 ur deuvieme tiers aval.

VIII. - Répartition de L'harmonique 3 Le Long DE LA CONDUITE.

Dans cerlaines conduites industrielles, nous avons remarqué, avec M. Eydoux, l'existence de l'harmonique 3.

La répartition de cet harmonique est évidente, il présente un noud en $A$ et en $C$,et un ventre en $B$ et en $D$.

La pression au deuxième liers aval $B$ sera conslante et égale à $y_{0}$ dans le cas d'unc conduite horizontale.

Les formules l'indiquent également.

On a en $\mathrm{B}$ :

$$
y=y_{0}+\mathrm{F}\left(-\frac{l}{3 a}\right)-\mathrm{F}\left(t-\frac{2 l}{a}+\frac{l}{3 a}\right)=y_{0} .
$$

puisque la fonction $\mathrm{F}$ a $\frac{4 l}{3 a}$ comme période.

Le cliché i6 représente l'inscription. de la pression au deuxième tiers aval an moment de la résonance de l'harmonique 3 .

Prenons le premier liers amont C. Les surpressions en A el G scronl décalées d'une demi-période, c'est-à-dire conslamment égales el de signes contraires.

La pression en $\Lambda$ est :

en $\mathrm{G}$ :

$$
y_{\mathrm{A}}=-y_{0}+\mathrm{F}(t)-\mathrm{F}\left(t-\frac{2 l}{a}\right)
$$

Or`:

$$
y_{0}=y_{0}+\mathrm{F}\left(t-\frac{2}{3 a}\right)-\mathrm{F}\left(t-\frac{2 l}{a}+\frac{2 l}{3 a}\right) \text {. }
$$

$\left.\mathrm{F}(t)-\mathrm{F}\left(t-\frac{2 l}{a}\right)=-\mathrm{F}(t) \frac{2 l}{3 a}\right)+\mathrm{F}\left(t-\frac{2 l}{a}+\frac{2 l}{3 a}\right)$,

toujours pour la même raison.

Donc :

$$
\left\{\begin{array}{l}
y_{\mathrm{A}}=y_{0}+\delta . \\
y_{\mathrm{c}}=y_{0}-\delta .
\end{array}\right.
$$

Pendant la résonance de l'harmonique 3 , l'amplitude de la surpression est donc la même au premier tiers amonl of à l'extrémité aval de la conduite.

Le décalage d'une demi-période des pressions en A el C se vérifie aisément en observant, au moment de la résonance de l'harmonique 3 , les équipages mobiles des deux mano. mètres placés en $A$ et $G$; c'est même un procédé qui permet de régler avec précision la vitesse du robinet tournant, pour obtenir la résonance de cel harmonique.

Le cliché $\mathrm{I} 7$ représente l'inscription de la pression en C au premier tiers amont. La pression est scnsiblement doublée par la résonance comme à l'extrémilé aval.

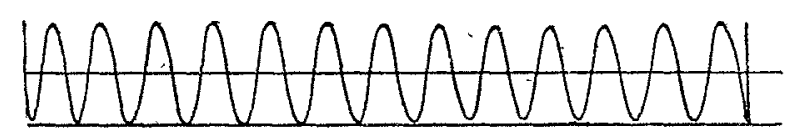

Fig. I7. - Harmonique 3; premier tiers amont.

J'altire l'attention des industriels sur le cliché 17 qu montre la nécessitè du renforcement des conduites au promiers tiers amont, lorsque l'harmonique 3 a une tendance marquée à se produire. Il ne faudrait pas croire d'aillcur's que les harmoniques ne se produisent que sous l'influence des régulateurs des turbines, ils peuvent prendre naissance spontanément dans des fermetures ; quand celles-ci ne sonl que partielles, le grand amortissement supprime tout danger, il n'en est pas de même à la fin de la fermeture. C'est là encorc un point sur lequel je me propose de revenir ultérieurement.
(A suivre.)
C Camichel,

Professeur à la Facullé des Sciences, Direcleur de l'Insitulut électrotechnique de Toulouse.

\section{LES FOURS A INDUGTION ET LEUR APPLication dans l'industrie du fer et de l'acier}

\section{CHAPITRE PREMIER \\ Apergu irstorique}

Lcs fours à induction employés jusqu'à présent dans l'industrie sont des transformateurs dont le sccondaire est formé d'une scule spire en court-circuil, dans laquelle la densité de courant est telle que le conducteur qui forme la spire est maintenu à l'ćlat de fusion. 
Le premier projet d'emploi industricl de ces fours remonte au brevel anglais, Ziani de Ferranti, numéro 700 de l'annee $188_{7}$. Suns parler, pour te moment, de l'agencement au poinl de vue électrïque des fours proposés par Ferranli, jo rais résumer brièvement el représenter par des croquis schómatiques les modes de production de la chaleur qu'll voulail appliquer Pour ec qui est des positions relatives du circuil magnélique, de la bobine primaire el du canal ammulaire de fusion, j'adoplerai, dans ces croquis, le type le plus usucl, celui des fours Kjellin.

Comvenons de plus que la signification des hàchures sera la suivante :

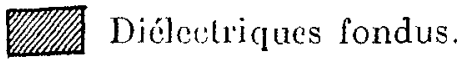

Conducteurs.

Dićleclriques infusibles.

Circuil magnétique.

Gela posé, yoici quels étaient les procédés considérés par Ferranti.

1. Echauffement de corps non conducteurs à l'élat froid remplissant des canaux on matière conductrice (figure. I).

2. Echauffement d'un conducteur remplissant un canal construil en matière infusible non conductrice à l'état froid (ligure 2).

3. Echauflement par le moyen d'un noyau en matière ronduclrice d'un corps non conducteur remplissant le canal de fusion (figure 3 ).

4. Echauffement de couches alternées de matières conductrices et non conductrices, remplissant le canal de fusion.

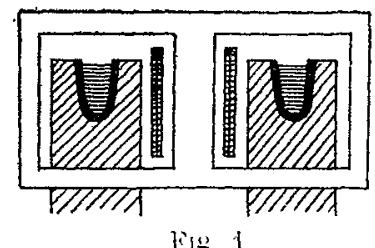

fis 1

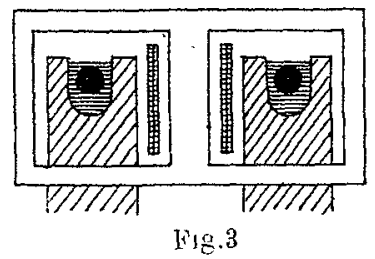

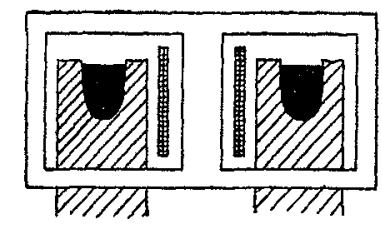

Fis, ?

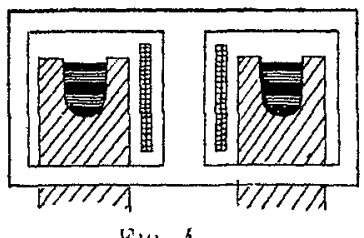

Fis 4
Par ce brevel de Ferranli, les divers procédés d'échauflenent des fouşs à induclion étaient défintivement fixés. Que ce brevel n'all pas, à son époque, attiré l'attention et n'ait pas cu d'application pratique, cola tient probablement au peu de développement qu'avait alors la technique des couranls altomalifs et au défaut de sources d'électricité à bon marché dans les contres industriels. Le brevet de Ferranti ne ful liré de l'oublı que lorsque d'aulres constructeurs curent oblenu des résultats pratiques. Mais étant donné la généralité des conceptions du brevet Ferranti, les projets utéricurs, dont quécques-uns, arrivèrent à une réalisalion industriclle, ne purent viser que des modifications dans la construction des fours ou la jecherche de donnécs électri yues micus appropriées.

Vers la fin de l'année r880, l'américain Dewey fit le pirojot d'échauffer, par induction, des cercles de roues et do les dilater ainsi. Vint cnsuite, en 1890 , Colby avec un bievel égalcment américain, pour un four a induction ; ef lorsqu'enfin le Suédois Kjellin eût construil à Gysinge, en r 899 , le premier four à induction pour l'obtention de l'acier et l'eûl amené dans les années suivantes à être utilisable indústriellement, alors les brevcts les plus divcrs relatifs à ces fours se succédèrent rapidement. Arant tout, signalons que tous ces fours appliquent le deuxième procédé d'échautfement, ci-dessus exposé (corps conductcurs remplissant un canal en matière conductrice) el visent presque exchusivement la produclion du fer el de l'acier, encore que les termes des brevels revendiquent des applications plus étendues.

\section{CHAPITRE II}

\section{Classirication des folrs a INDUC'ION D'Aphes LEUR AGENCEMUNT ĹLECTRTQUE.}

Les principarıx types de fours à induction actucllement connus se divisent en deux groupes à savoir :

Fours à induction pure. - Dans lesquels la matière à fondre est réparte dans tout l'anneau secondaire et n'est échauffée que par induction, et fours mixtes dans lesquels on a ajouté une matıère résistante infusible qui est directement échauffée par le courant primaire ou qui constitue une partie du circuit secondaire.

Il est à remarquer par ailleurs que dans les opérations sidérurgiques - qui sont évidemment les plus importantes à considérer, - le métal liquide est couvert d'une couche plus ou moins épaisse de scories c'est-ầ-dire de substances chimiques (silicates) qui se comportent comme des conducteurs de la scconde classe. Or les deux conducteurs, le métal et la scorie, peuvent dans les fours être placés en parallèle ou en sérje. Il y a là le principe d'une nouvelle subdivision.

\section{$\S \mathrm{x}$. - Fours à induction pure.}

A. Métal et scorie sont en parallèle. - Le courant secondaire se partage entre les deux conducteurs en raison de leur conductibilsté. Dans ce type le canal de fusion est sensiblement horizontal el contient done dans toute son élendue deux couches liquides non miscibles.

a Le métal liquide a dans toute l'étendue du canal la même section, abstraction faite des petiles varialions qui pourraient résulter d'une usure irrégulière des revêtements du four dans ses différentes parlics. La lenjpéralure du bain sera alors uniforme.

La bobine primaire est cylindrique. Le four correspond alors à un transformateur à enroulements cylindriques.

r) Four de Colby, I8go. (Fig. 5). - I a bobine primaire et le canal de fusion sont concentriques, la bobine entourant le canal. Le circuit magnétique conslitue deux anneaux accolés.
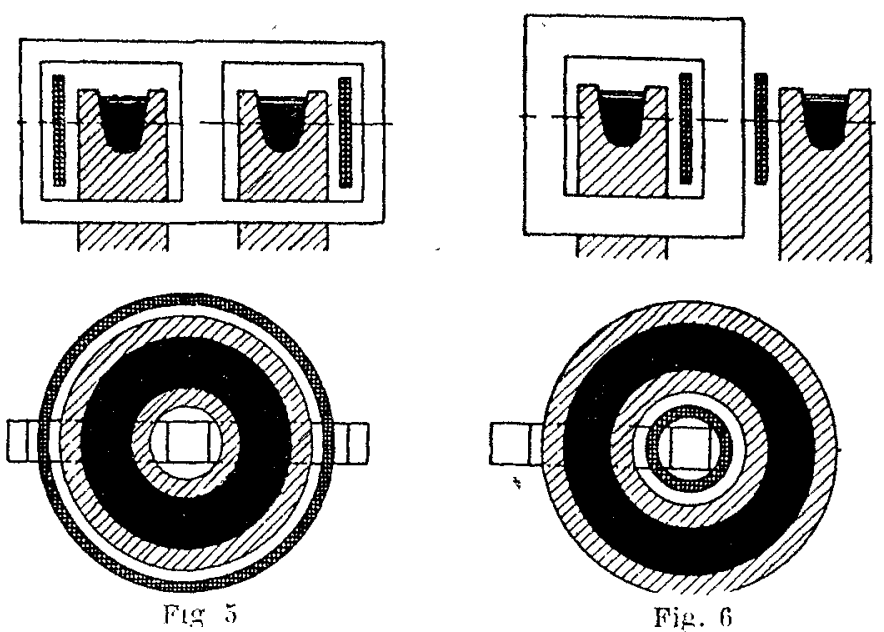

2) Four de Kjellin, rgoo (Fig. 6). - la bobinc primaire et le canal de fusion sont concentriques. Le canal entoure la bobine. Le circuit magnélique forme une simple boucle. 
3) Four de Kjellin, rgo5. (Fig. 7). - Ia scule différence avec le précédent c'est que le circuit magnélique forme une double boucle.

4) Four de Mjorth, Igo5. (Fig. 8). - La bobine primaire el le canal sont extérieurs l'un à l'autre. I ce circuit magnétique est un simple anneau. Dans ce four il a'y a que le noyau embrassé par la bobine primaire qui soi, fire : Je reste de larmature peut ètre fixé alternativenent à gauche of ì droite, pour pouvoir opérer avec un mêne transformaleur sur deux canaux de fusion différents.


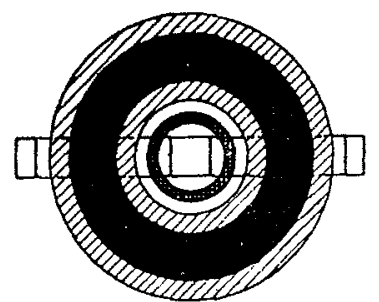

Fig. 7

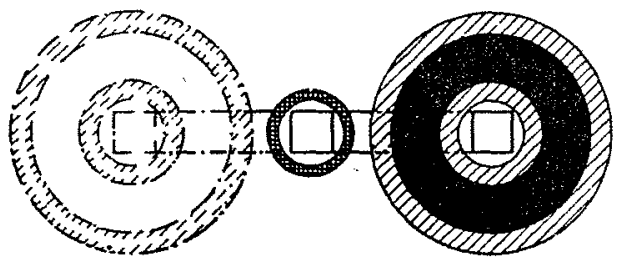

Fig, 8
B. La bobine primaire est en forme de disque. - Le four correspond à un transformaleur à enroule ane uts ext disques.

5) Four de Ferranti, I887. (Fig. 9). - Les bobines plates primaires sont disposées en dessus et en dessous du canal de fusion. Le circuit magnétique forme un double anneau.

6) Four de Frick, Igo4. (Fig. ro). - La bobine plate primaire est au-dessus ou bien au-dessous du canal de fusion. Le circuit magnétique forme un simple annear.
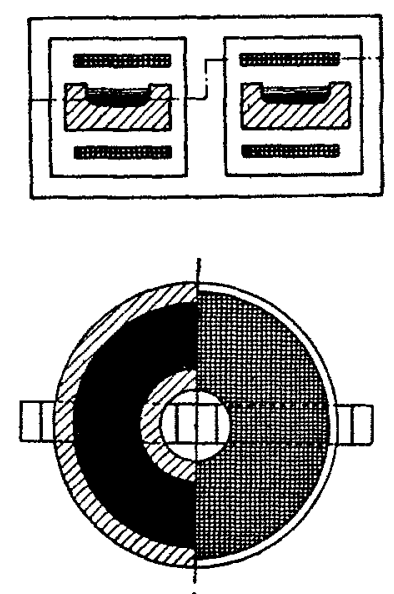

Fis 9

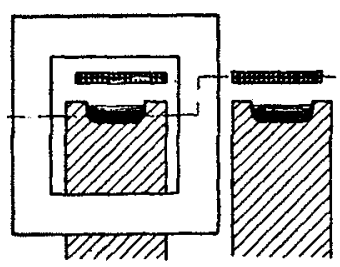

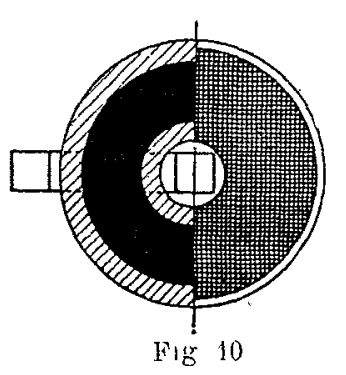

b. Le mélal liquide nemplissant le canal a des sections différentes dans les différentes parties du canal. La raison d'être de cette disposition est d'obtenir un meilleur brassage du métal fondu qui s'échaufle plus là où la section est plus laible ce qui lc porte à monter à la surface el produit dans le canal une circulation du métal dans un sens prérí ì lavance.

7) Four de Schneider au Creusot, Igo3. (Fig.xx). - Le canal de fision sc compose d'un large réservoir el d'un conduil anmulaire en forme d'anse. Le réscrvoir est ouvert i l'air libre, le conduit ne l'est pas. Le métal plus chaud doit constamment monter de celle anse dans le réservoir cl provoquer ainsi des courants de convection. Le primaire cst en duhors du canal de fusion. L'enroulement est de forme cylindrique.

8) Foup de Gin, 19o6. (Fig. 12). - I.c canal de fusion se compose de deux larges réservoirs rectangulaires dont les fonds sont inclinés en sens inverses el reliés par des conduits ammulaires inclinés eux aussi, de sorte que l'ensemble forme quatre canaux en zig-zag. La bobine primaire el le canal de fusion sonl concenlriques. Le circuil magnétique forme $\mathrm{m}$ double amean. lienroulement primaire est cylindrique. A part la forme du canal ce four reproduit alui de Kjellon, I $90 . \overline{7}$.
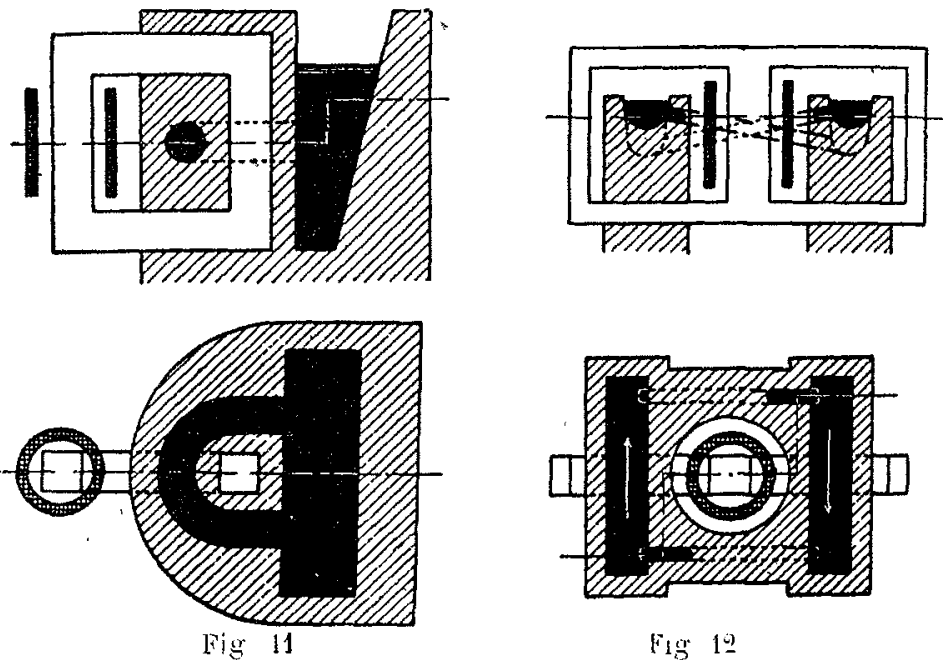

Fig 19

B. Le métal ef la scorie sont en série. Le canal de fusion est verlical et le couranl secondaire passe en mêrne intensilé à travers le métal el à trẫers la scorie. Une partie du canal de fusion n'est remplie quavec du métal, l'autre n'est remplie qu'avec de la scorje.
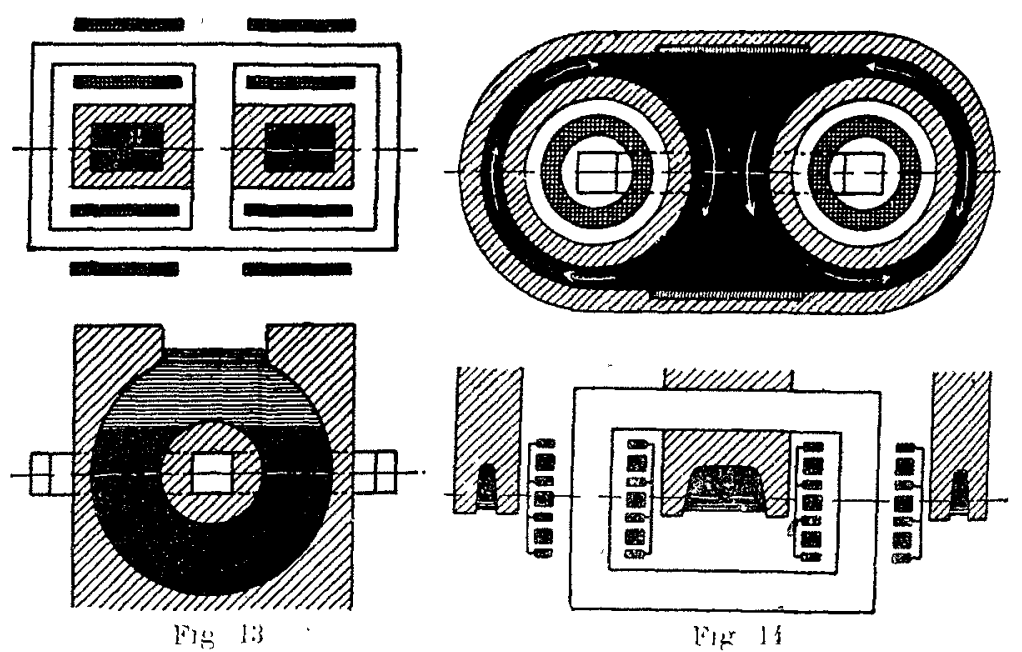

9. Four de Wallin, rgo4 (figure r 3). La chambre de lusion se compose d'un canal annulaire, construit verlicalement dans la maçonnerie. I.e chargement se fait à travers la couche de scorie. L'enroulement primaire se composc de quatre cylindres horizontaux.

\section{FOURS MIXTES}

ro. Fou'x de Röchling-Rodenhauser, rgo5 (Ggure 14). Le sccondaire se compose de drux parlies : d'une part, deux canaux de fusion en corrt-circuit avec foyer commun : d'autre part, des spires conductrices en série avec certaines partics du revêlcment du four construiles en malière conductrice. On réchaufle ainsi encore plus le foyer commun.

I I. Four de Hjorth, rgo6 (figure r5). Ce four correspond pour la disposition du primajre, du noyau, el du canal de 
fusion au promicr four de IJjorth (1905). Le canal de fusion est cependant inlerrompu par une paroi infusible, enjambéc elle-même par une électrode donl les extrémités ne haigncnt que dans la scorie. C'est, en somme, une combinaison du four lléroull à électrodes el du four à induction
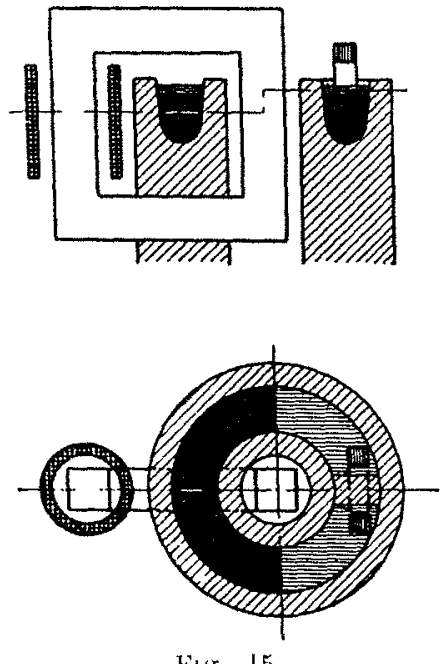

Fis. 15

12. Four de Iljorth, 1906 (figure I6). Le secondaire est constitué par une électrode en forme d'U renversé, qui ne plonge encore que dans la scorie. Combinaison encore d'un fout Héroult et d'un four à induction.
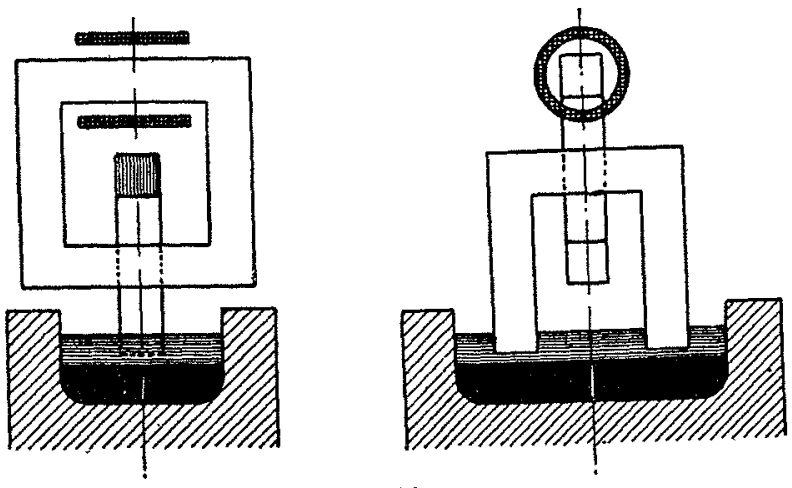

Fin 16

CHAPITRE III

ExigeNGes AUXQLeldes Les fours a INDUCTION DOIVENT SATISFAIRE

Il y a trois conditrons principales el parfois incompatibles, à réaliser :

$I^{\circ}$ Réduire au minimum la dépense d'énergie électrique ;

$2^{\circ}$ Utiliscr le micux possible la chaleur produite;

$3^{\circ}$ Faciliter les opérations métallurgiques.

Au point de vue électrique, ce qui importe le plus, c'est de diminuer les fuiles en rapprochant le primaire et le sccondaire. En principe, cela pourrait être obtenu aussi bien avec un enroulement en disques qu'avec un enroulement cylindrique. Il faut donc faire intervenir d'autres considérations pour pouvoir établir un parallèle entre ces deux enroulcments.

Enroulement cylindrique. - Dans l'enroulement cylindrique, pour avoir une moindre dispersion, on mettra le primairc à l'intérieur du canal de fusion et on fera celui-ci profond et étroit. On sera arrèté dans celte voie par la nécessité de pouvoir charger le canal avec des matériaux solides d'une certaine grosseur. De plus, la profondeur du bain angmente la pression sur les parois et le risque d'une rupture de celles-ci par le métal en fusion. Enfin, si l'accroissement de profondeur du bain et la diminution de surface lıbre qui en résulte réduisent les pertes par rayonnement, en revanche l'extension donnée à la surface de contact rend plus considérables les pertes par conductibilité.

Ie rapprochement du primaire et du secondaire est limité également par la nécessité de donner à la paroi du canal de fusion, une solidité suffisante pour résister à la poussée du métal. Par aillcurs, il faut entre le primaire et le secondaire, disposer des appareils de réfrigération par eau ou par air, alin de proléger contre la chaleur la bobine primaire et le noyau.

Je signalerai, par contre, un avantage indiscutable de l'enroulement cylindrique, à savoir que le canal de fusion est facilement accessible.

Enroulement en disques. - L'enroulement en disques ne devient avantageux qu'avec un canal de fusion large et peu profond et avec une bobine primaire formant un disque de mêmes dimensions générales que le canal. Mais il faut distinguer deux cas suivanl que la bobine est en dessus ou en dessous du bain.

Si elle est en dessus, on pourra la rapprocher aulant qu'on voudra du métal en fusion, mais cela gênera et rendra peutêtre impossible le chargencut. Et la bobine primaire sera exposée à des avaries qui, avec les grands modèles de fours à induction, seront d'aulant plus à redouter qu'il y a des mourements tumultueux dans la masse en fusion. D'autre part si, pour utiliser complètement les avantages des enroulemenls en disques, on fait un canal large et aplati, on augmente les pertes par rayomement et la surface de contact du mélal fondu avec le revêtement, ce qui rend plus importante l'usure du revêtcment.

$\mathrm{Si}$, au contraire, on met la bobine primaire en dessous du canal de fusion, la maçonnerie du cà nol empêch. de réduire au-delà d'unc certaine limite l'intervalle entre primaire et secondaire. Sans compler que la bobine courra un plus grand danger d'être détériorée par des éclaboussures de métal lrquide. D'autre part, Je canal étant encore large et aplati, les pertes par rayonnoment ne sont pas diminuées.

Ce qui ressort le plus clairement de ce parallèle entre les deux types d'enroulements, c'est qu'il faut loujours établir un compromis entre le métallurgiste et l'électricien.

Tension prinaire. - Un avantage des fours à induction de loul système, c'est qu'on peut choisir arbitrairement la tension primaire sur une chelle très étendue. Si l'on fail abstraction de la question de sécurité du personnel, il s'agit seulement de sarorr combien, en lenant comple des particularilés locales, on veut immobiliser de cuivre dans le primairc sans faire renchérn à l'excès l'installation, ni accrô̂tre pas trop le diamètre du canal.

Masse du chargement - Ce qui influe grandement aussi sur les données électricues, c'est la masse du chargement. Sans doute, les fours Kjellin de huil tonnes, sont déjà d'une grandeur notable, mais il y a tendance à faire de plus grandes unités encore, surtont dans les instalfations adjointes à des fours Thomas ou Martin-Siemens, afin de pouvoir mettre dâns le four éleclrique le chargement entier du convertisseur ou du four à llammes.

Avec l'augmentation du chargement, la résistance ohmique du secondaire diminue, mais sa self induction augmentc, ce qui amoindrit Is facteur de puissance. Kjellin et Röchling-Rodenhauser ont paré à cet inconvénient par deux méthodes différentes.

Kjellin diminue la fréquence à mesure que la grandeur du four augmente. Cela nécessite naturellement un géné- 
rateur spécial qui revient plus cher qu'une machine normale. Mais, comme le choix d'une faible fréquence ne s'impose que pour de très grosses unités (cinq périodes pour r.ooo (hevaux) qui justifient l'installation d'un génćratcur spécial, l'augmentation du prix d'achat n'intervient que pour majorer les frais d'amortissement, ce qui ne doit pas entrer en considération, vu le débit énorme de ces fours. Cependant, il serait désirable de pouvoir brancher le primaire d'un four sur un résean à fréquence normale, surtout pour les petils fours deslinés à des usages spéciaux, comme la fonderic de bronze.

Dans ce sens, Röchling-Rodonhauser a réalisé un progrès considérable en ajoutant au four Kjollin un dispositif de chauffage par résistances. D'une part, le canal en forme de huit enveloppanl les deux noyaux, cela permel de réduire la section du bain et d'augmenter sa résistance ohmique. Même avec des chargements de grandeurs croissantes, il n'est pas utile d'angmenter dans la même proportion la section des deux boucles du canal de fusion, l'augmentation de volume se reportant principalcment sur le foyer central. D'autre part, le facteur de puissance est encorc augmenté par le fait qu'une part du courant secondaire circule dans les résistances de chaufrage du foyer central. Ces fours peuvent être alimentés à la fréquence 50 , même s'ils atteignent des dimensions très notables, et pour les plus grandes unités, il n'est pas nécessaire de dépasser la fréquence encorc usuelle de vingt-cinq périodes.

\section{(4 suivrè)}

\section{L'ÉLECTRIFICATION DE NOS RÉSEAUX FERRÉS}

Nous avions indiqué à nos lecteurs les efforts faits en vue de remplacer, avec toutes les chances de succès possible, la traction à vapeur par la traction électrique sur nos voies ferrées. Nous avions annoncé le départ d'une mission d'étude, sons la direction de M. Maudurt, Professeur à la Faculté des Sciences de Nancy, pour l'Amérique et nous avions promis de publier le résultat de l'enquite de celte mission : un comple rendu sommaire a été a iñessé au Ministre des Travaux publics et publié dans le Jo:rn. Offic. du I 3 août igrg. Nous le reproduisons ci-dessousin-extenso.

\section{Ministère des Trayaux publics, des Transports et de la Marine marciande}

\section{COMPTE RENDU SOMMAIRE}

adressé au Ministre des Travaux publics par la mission envoyée en Amérique pour l'étude de lélectrification des grands réseaux de chemins de fer.

Le ministre des Travaux publics a instilué, par arrêlé du Í novembre s 9 r 8 , au sein du Conseil supérieur des Travaux. publics, un comité d'études chargé d'examiner les projels présentés par Jos réseaux de Paris-Lyon-Méditerranée, d'OrJéans et du Midi, pom l'alectrification de 10.000 kilomètre chviron de lignes de leurs réseaux.

Ce coinilé, composé de techniciens les plus qualifiés de ladministration et des réseaux de chomins de fer, a cru devoir proposer au ministre d'envoyer aux Etats-Unis une mission d'ingénieurs spécialistes, chargée de recueillir toules les informations relatives aux progrès récents de la traction électrique.

\section{$x^{\circ}$. - Onganisation et composition de la mission}

Cette mission comprenail treize membres, savoir :

Commandant d'Adglards et professeur A. Mauduit, de la Faculté des Scicnces de Nancy, attaché à la direction des chemins de fer, délégués du ministère des travaux publics et des transports :

MM. Pomey, ingénicur en chef des posles el dos télégraphes et Lecorbciller, ingénicur, délćgués de l'administration des postes et des télégraphes ;

MM. Debray, inspecteur, principal, et Barillot, inspecteur, délégués des chemins de fer de l'Etat;

M. Sabouret, ingénicur en chef adjoint à la dircction ;

MM. Balling, ingćnieur principal au service de la voie et Parodi, ingénieur, chef du service électrique, délégués de la compagnie des chemins de fer d'Orléans ;

MM. Japiot, ingénieur en chef du matéricl ol Ferrand, ingénieur au service central du matériel, délégués do la compagnie Paris-Lyon-Médilirranée ;

MM. Bachellery, ingénieur en chef adjoint à la direction, et Leboucher, ingénieur principal à la traction, délégués do la compagnie des chemins de fer du Midi.

La plupart des membres de la mission sont partis le I5 avril pour l'Amérique et sont rentrés à Paris, lc 22 juillet rgrg.

\section{$2^{\circ}$. - Itinératre ex trayaUt de la mission}

Arrivés à New-York, le 25 avril, nous avous pris contact avec les représentants des diverses usines de construction el compagnics de chemuns de fer et visité les chemins de fer électrifiés suivants :

New-York central, courant conlinu à 600 volts, $3^{e}$ rail ;

New-York New-Haven and Hartford, monophasé à I I.000 volts, 25 périodes;

Pensylvania railroad et Long Island $R$. $R$. continu 600 volts, $3^{\circ}$ rail ;

Chemins de fer de banlieue, assurant cependant un trafic appréciable de marchandises.

Nous avons également visité un certain nombre de centrales électriques puissantes à vapeur, de la Interborough Transit $\mathrm{C}^{\circ}$ et de la Edison Power $\mathrm{C}^{\circ}$ à New-York, ainsi que les centrales du Niagara (hydrauliques) et de Buffalo (à vapeur).

Du 8 au ro mai, visite des atcliers de la General Electric Company (G. E. $\left.\mathrm{C}^{\circ}\right)$ à Schenectady (N. Y.) et discussion avec les principaux ingénjeurs de cette société, sur les questions concernant l'élcctrification des chemins de fer, en génćral, et notamment l'électrification en courant continu à haulc tension (3.000 volts) du Chicago Milwankee Saint-Paul (7ro kilomèlres en fonctionnement), exécutée par celle société.

Du ix au 25 mai, visite des inslallations suivantes

Electrification du Norfolk and Western railway, en colrant monolriphasé, à I $\mathrm{I}$.ooo volts-25 périodes, de Bluefield à Vivian (Virginie).

Electrification du Pensylvania raijroad, en monophrsí $x$ x.000 volts, 25 périodes, de Philadelphie à Paoli..

Chemin de fer électrique Baltimore-Woshinglon-Annapolis, en courant continu r.20o volts (interurbain).

Ateliers de construction de locomolives Baldwin à Philitdelphie.

Ateliers de réparation et de construction du Pensylvania railroad à Altona.

Du 25 au 28 mai, visile des aleliers de la socićté de construction Westinghouse électric and manufacturing company, 